\title{
Laser-induced white-light emission from graphene ceramics-opening a band gap in graphene
}

\author{
Wieslaw Strek ${ }^{1}$, Bartlomiej Cichy ${ }^{1}$, Lukasz Radosinski ${ }^{2}$, Pawel Gluchowski ${ }^{1}$, Lukasz Marciniak ${ }^{1}$, \\ Mikolaj Lukaszewicz ${ }^{1}$ and Dariusz Hreniak ${ }^{1}$
}

Recent theoretical and experimental studies have indicated the existence of a new stable phase of carbon with mixed $\mathrm{sp}^{2}$ and $\mathrm{sp}^{3}$ hybridized bonds-diaphite. Such a two-layered structure with $\mathrm{sp}^{2} / \mathrm{sp}^{3}$ bonds may be observed after the photostimulation of highly oriented pyrolytic graphene with femtosecond laser pulses. This hidden multistability of graphene may be used to create a semiconducting phase immersed in the semimetallic continuum, resulting in bandgap opening. We demonstrate that bandgap opening and light emission from graphene is possible using continuous-wave laser beams with wavelengths from the visible $(405 \mathrm{~nm})$ to the near-infrared range $(975 \mathrm{~nm})$. We demonstrate that without the application of cooling, the effective temperature of the emitting sample remains lower than $900 \mathrm{~K}$, which is far below the value predicted by the theory of black-body radiation. Moreover, light emission from a graphene sample may be observed at temperatures as low as $10 \mathrm{~K}$.

Light: Science \& Applications (2015) 4, e237; doi:10.1038/Isa.2015.10; published online 16 January 2015

Keywords: ceramics; graphene; photoluminescence; white light

\section{INTRODUCTION}

Because of its exceptional properties, graphene is the focus of many extensive investigations. The two-dimensional crystal structure, linear energy dispersion and zero optical band gap of graphene, along with the presence of massless Dirac quasiparticles, have successfully focused considerable scientific attention on this material, which theoretically should not exist. ${ }^{1}$ A number of studies have already reported many excellent applications of graphene in various fields, e.g., new types of field-effect transistors, ${ }^{2}$ composite conductors ${ }^{3}$ and sensor applications. ${ }^{4,5}$

In addition to the interest in pristine graphene, considerable scientific attention has also been devoted to the chemically derived hybrid known as graphene oxide, which exhibits unusual and tunable properties. $^{6}$ As a chemical derivative, graphene oxide possesses distinctly different properties than those of pristine graphene, which originate from the particular characteristics of the chemically reactive functional groups, e.g., hydroxyl, epoxide, carbonyl and carboxyl groups, bound to the surface of grapheme. ${ }^{7}$ By controlling the $\mathrm{sp}^{2} / \mathrm{sp}^{3}$-hybridization ratio and the nature of the reactive groups, one is able to control the optical and electrical properties of the resulting material. ${ }^{6}$

Recently, considerable scientific interest has also been devoted to studies of the white-light (WL) emission from carbonaceous materials, using laser light or an electrical current as an excitation source. Incandescence experiments have been performed on a number of carbon structures, including carbon nanotubes, ${ }^{8}$ fullerenes ${ }^{9,10}$ and graphene, ${ }^{11,12}$ which are being considered as materials for next-generation light sources. In particular, carbon nanotubes have been investigated for their potential application as chemically stable thermal filaments in next-generation incandescent sources. ${ }^{13}$ However, although considerable efforts have been made in the development of carbon-based devices, ${ }^{14}$ discussion of the WL emission from graphene is still severely lacking.

It has previously been reported that pristine graphene exhibits no measurable light emission under continuous-wave (CW) laser excitation; however, weak emission following the model of thermalized emission has been observed under femtosecond pulse excitation. ${ }^{15}$

The theory of black-body radiation is naturally the first choice for the interpretation of the origin of light emissions from graphene and other carbonaceous materials. ${ }^{16}$ Various models have been proposed to explain the origins of laser-induced incandescence, which is a complex phenomenon that involves laser absorption with the simultaneous emission of WL, which is responsible for cooling the irradiated material. ${ }^{17}$ The observation of visible electroluminescence from multilayer graphene systems has also been reported by several authors. ${ }^{18}$ Biswas et al. ${ }^{19}$ have reported tunable electroluminescence from planar graphene $/ \mathrm{SiO}_{2}$ structures. Dong et al. ${ }^{20}$ have reported blue luminescence with a high quantum yield. Current-induced incandescent light emission has also been observed in large-area graphene films by $\mathrm{Yu}$ and Dai. ${ }^{13}$ Recent studies of confined graphene systems have demonstrated the production of laser-excited WL emission from edge-functionalized graphene quantum dots. ${ }^{21}$

To the best of our knowledge, we report here, for the first time, laser-induced WL emission from cooled $(10 \mathrm{~K})$ and moderate-temperature $(<900 \mathrm{~K})$ graphene ceramics. The purpose of this work was to investigate the WL emission from graphene ceramics with regard to

${ }^{1}$ Polish Academy of Sciences, Institute of Low Temperatures and Structural Research, 50-422 Wroclaw, Poland and ${ }^{2}$ Wroclaw University of Technology, Group of Chemical and Biochemical Processes, 50-370 Wroclaw, Poland

Correspondence: B Cichy, Polish Academy of Sciences, Institute of Low Temperatures and Structural Research, Okolna 2, 50-422 Wroclaw, Poland

E-mail: b.cichy@int.pan.wroc.pl

Received 16 July 2014; revised 31 October 2014; accepted 4 November 2014; accepted article preview online 19 November 2014 
the potential application of these materials as alternative WL sources. The distinctive features of this WL emission include the independence of the emission spectra with respect to the ambient temperature between $10 \mathrm{~K}$ and $300 \mathrm{~K}$; the spectral independence of the emission band with respect to the excitation wavelength, which results in Stokes and anti-Stokes emission; the nonlinear dependence of the process; and the wavelength-dependent power threshold. We demonstrate that without cooling, the effective temperature of the emitting sample remains lower than $900 \mathrm{~K}$, which is far below the value predicted by the theory of black-body radiation. Moreover, it is shown that laserinduced WL emission from graphene ceramics is also clearly observed at cryogenic temperatures as low as $10 \mathrm{~K}$. The results are discussed in terms of transient photoinduced domain-like phase conversion in disordered graphene systems.

\section{MATERIALS AND METHODS}

Preparation of the graphene ceramics

The graphene ceramics (GCs) were prepared from commercial (CheapTubes.com. Cambridgeport, VT, USA) graphene nanoplatelets. The graphene nanoplatelets were characterized by surface areas of $600-750 \mathrm{~m}^{2} \mathrm{~g}^{-1}$, the presence of 4-5 graphene layers with an average thickness of $8 \mathrm{~nm}$, and a single-particle diameter of less than $2 \mu \mathrm{m}$ (see Supplementary Information for transmission electron microscopy images of the as-received graphene nanoplatelets).

The GCs were fabricated in the form of pellets using the hot isostatic pressing method, which requires both an elevated temperature and an isostatic pressure. As a first step, the graphene flakes were formed into pellets of $6 \mathrm{~mm}$ in diameter and $2 \mathrm{~mm}$ in thickness via cold pressing at $0.2 \mathrm{GPa}$. The as-formed pellets were then placed in a calcium carbonate $\left(\mathrm{CaCO}_{3}\right)$ toroid and separated from the heating element by a thin layer of boron nitride. Each sample was then pressed at $8 \mathrm{GPa}$ and heated to $500{ }^{\circ} \mathrm{C}$ for $1 \mathrm{~min}$. As a final step, each GC sample was mechanically polished to remove the residual boron nitride powder and the topmost layers of graphene. Afterward, the sample was placed in a vacuum chamber (see Supplementary Information for scanning electronic microscopy images of the GCs).

\section{Synthesis of $\mathrm{LiYb}_{0.99} \mathrm{Er}_{0.01} \mathrm{P}_{4} \mathrm{O}_{12}$ nanothermometers}

Erbium $\left(\mathrm{Er}^{3+}\right)$-doped lithium ytterbium tetraphosphate nanocrystals were prepared via a wet chemistry route. The starting materials for the synthesis were used without additional purification and were as follows: $\left(\mathrm{NH}_{4}\right)_{2} \mathrm{HPO}_{4}$ (POCH, 99.99\%), erbium(III) oxide $\left(\mathrm{Er}_{2} \mathrm{O}_{3}, 99.99 \%\right.$; Sigma-Aldrich, Poland), ytterbium(III) oxide $\left(\mathrm{Yb}_{2} \mathrm{O}_{3}, 99.99 \%\right.$; SigmaAldrich) and $\mathrm{Li}_{2} \mathrm{CO}_{3}$ (POCH, 99.99\%). Lanthanide ions were added in the form of nitrates after prior dissolution of the corresponding oxides, e.g., $\mathrm{Yb}_{2} \mathrm{O}_{3}$ and $\mathrm{Er}_{2} \mathrm{O}_{3}$ were dissolved in concentrated nitric acid $\left(\mathrm{HNO}_{3}\right)$. The $\left(\mathrm{NH}_{4}\right)_{2} \mathrm{HPO}_{4}$ was dissolved in deionized water and subsequently mixed with $\mathrm{Li}_{2} \mathrm{CO}_{3}$ and lanthanide nitrates containing appropriate amounts of $\mathrm{Er}^{3+}$ and $\mathrm{Yb}^{3+}$, resulting in a homogeneous solution. The $\mathrm{LiYb}_{0.99} \mathrm{Er}_{0.01} \mathrm{P}_{4} \mathrm{O}_{12}$ nanocrystals were obtained after the precipitation and transformation of the amorphous product into a crystalline product via thermal treatment.

The structural properties of the graphene flakes were examined via high-resolution transmission electron microscopy using a Philips CM20 SuperTwin transmission electron microscope, which provides a resolution of $0.25 \mathrm{~nm}$ at $200 \mathrm{kV}$ (see Supplementary Information).

The luminescence spectra were measured using an Avantes AVSUSB2000 CCD spectrometer and a number of excitation laser sources, from the visible (VIS) up to the near-infrared (NIR) regime: $\lambda_{\text {exc }}=975 \mathrm{~nm}, 808 \mathrm{~nm}, 514 \mathrm{~nm}, 488 \mathrm{~nm}, 476 \mathrm{~nm}$ and $405 \mathrm{~nm}$. All graphene ceramics were measured in vacuum $\left(10^{-3} \mathrm{hPa}\right)$ and excited by a laser light focused to a spot size of $1 \mathrm{~mm}$.

The temperature of the graphene ceramics was determined using $\mathrm{LiYb}_{0.99} \mathrm{Er}_{0.01} \mathrm{P}_{4} \mathrm{O}_{12}$ nanocrystals encrusted on the ceramic surface.

The photoconductivity of the graphene ceramics was measured via the four-point method using four gold electrodes fixed with silver conducting paste. A 10-mA current source (Keithley 2002) was used in the experiment to measure the voltage drop across the sample.

\section{RESULTS AND DISCUSSION}

The WL emission from the graphene ceramics was measured in oilfree vacuum $\left(5 \times 10^{-3} \mathrm{hPa}\right)$. The samples were excited by a CW laser beam focused to a spot of $1 \mathrm{~mm}$ in diameter. The laser-induced WL emission was investigated at a number of different excitation wavelengths, from the VIS up to the NIR range, i.e., at $476 \mathrm{~nm}, 488 \mathrm{~nm}$, $514 \mathrm{~nm}, 808 \mathrm{~nm}$ and $975 \mathrm{~nm}$. Representative spectra of the WL emission from the graphene ceramics upon irradiation at various excitation wavelengths are presented in Figure 1a.

There are several features of the data presented in Figure 1a that are worth noting. One distinctive feature of the data is its wide spectral range, extending over the VIS and NIR regions. Notably, the emission occurs at energies higher than the energy of the exciting photons. This suggests that cascade multiphoton processes must be involved in this emission. At least three characteristic maxima, at $1.97 \mathrm{eV}(629 \mathrm{~nm})$ and $1.83 \mathrm{eV}(677 \mathrm{~nm})$ and a shoulder at $1.68 \mathrm{eV}(738 \mathrm{~nm})$, may be identified in the emission band. It is worth noting that the presence of more than one band maximum is in conflict with the theory of blackbody radiation. A representative spectrum of black-body radiation for a temperature of $T=4315 \mathrm{~K}$ is also presented for comparison.

No significant dependence of the broadband WL emission maxima on the excitation wavelengths was observed (Figure 1a). The peak maxima are located at nearly identical positions for each wavelength, and only a slight broadening of the band is observed with decreasing excitation wavelength. The WL emission band is centered at approximately $1.87 \mathrm{eV}(660 \mathrm{~nm})$.

The chromaticity coordinates of the WL emission were calculated for all excitation wavelengths and are depicted in Figure 1b. Small shifts in the chromaticity coordinates were observed; however, all samples were located in the white-orange region. Moreover, no particularly strong shifts in the emission-band maxima were observed for different excitation powers; however, it was observed that WL emission from the graphene ceramics was a threshold process exhibiting supralinear behavior.

The dependence of the laser-induced WL emission on the excitation light power is illustrated in Figure 2. A representative plot of the WL emission excited at $975 \mathrm{~nm}$ for various laser powers is presented in Figure 2a. The nonlinear behavior of the excitation power, along with the threshold point, is shown in Figure $2 \mathrm{~b}$ for a number of excitation wavelengths. Once the threshold point is crossed, the WL emission increases in accordance with the power-law formula (Equation (1)):

$$
I_{\mathrm{WL}} \propto P^{N}
$$

where $I_{\mathrm{WL}}$ is the intensity of the WL emission, $P$ is the power of the excitation laser light and $N$ is the order of the process. A monotonic dependence of the order of the process on the excitation wavelength was observed (Figure 3). The lowest value of $N=2.39$ was observed for $\lambda_{\text {exc }}=405 \mathrm{~nm}$, and the highest value of $N=5.69$ was observed for $\lambda_{\mathrm{exc}}=975 \mathrm{~nm}$. For high-energy photons $(3.06 \mathrm{eV})$, the WL emission appears to behave like Stokes emission, whereas for lower-energy excitation, the emission may be treated as an anti-Stokes process. 


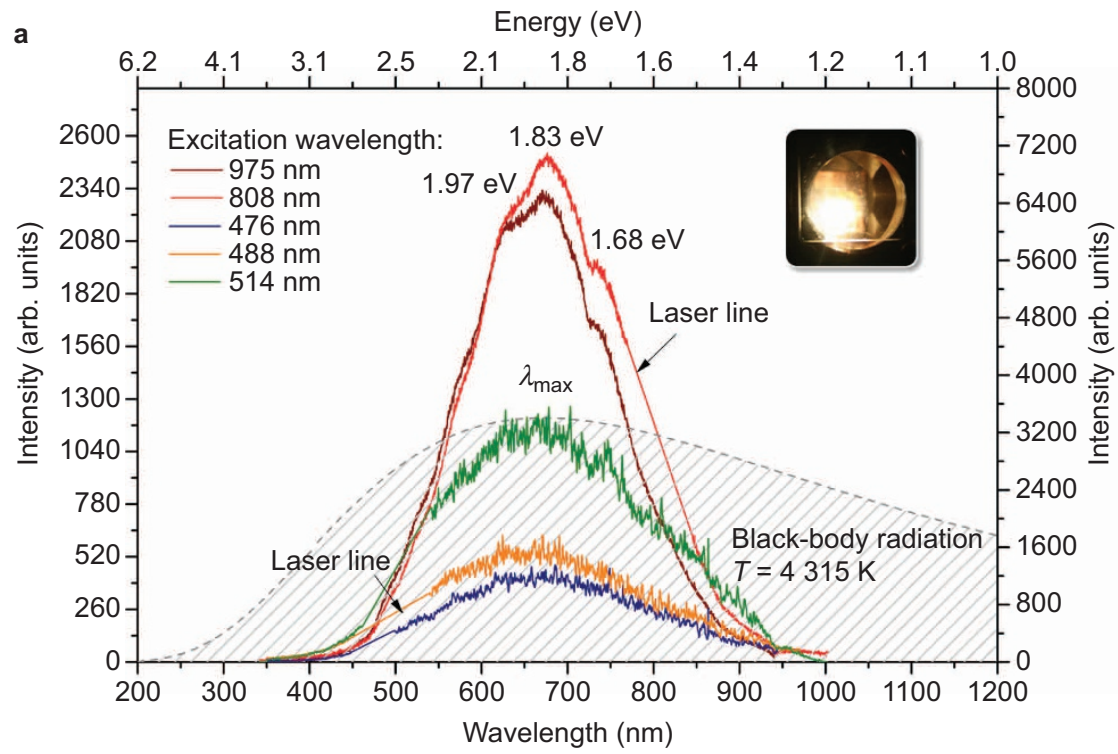

b

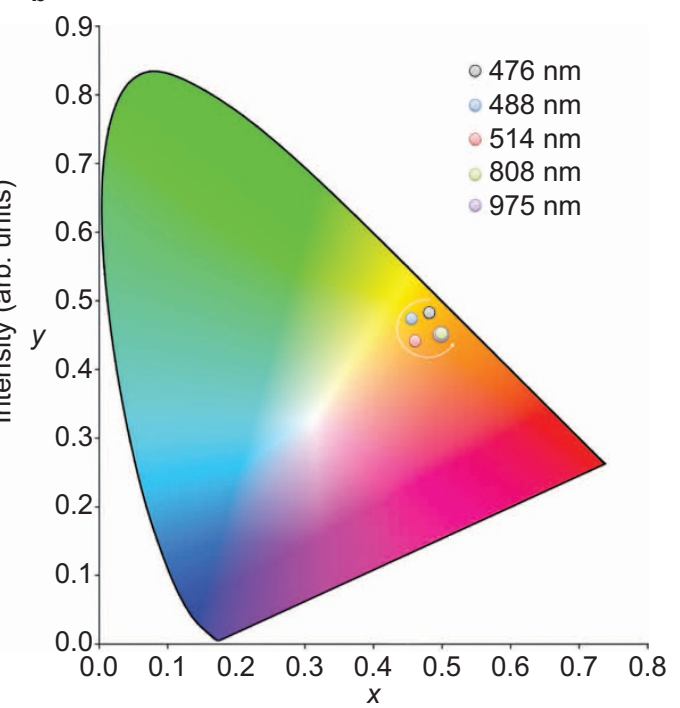

Figure 1 (a) Dispersion of the laser-induced light emitted from the graphene ceramics upon irradiation at various excitation wavelengths $\lambda_{\text {exc }}$ from the VIS to the NIR range, i.e., $476 \mathrm{~nm}, 488 \mathrm{~nm}, 514 \mathrm{~nm}, 808 \mathrm{~nm}$ and $975 \mathrm{~nm}$. All measurements were performed in vacuum $\left(<10^{-3} \mathrm{hPa}\right)$. The black-body radiation spectrum at $T=4315 \mathrm{~K}$ is provided for comparison. (b) Chromaticity diagram with coordinates for each excitation wavelength. The excitation laser lines were removed from the spectra, as indicated by an arrow on the graph. A representative image of the light emitted from the graphene ceramics is presented as an inset in a. NIR, near-infrared; VIS, visible.

This finding indicates that the WL emission from graphene ceramics has its origin in multiphoton absorption.

The total energy $E_{\text {tot }}$ of $N$ absorbed photons with energy $\hbar \omega_{\text {exc }}$ was nearly identical for all investigated excitation wavelengths, $E_{\text {tot }}=$ $N_{\text {exc }} \hbar \omega_{\text {exc }}$. If we take the photon energy corresponding to the $\lambda_{\text {exc }}=$ $975 \mathrm{~nm}$ excitation wavelength $(1.27 \mathrm{eV})$ and multiply it by a number of photons equal to 5.69 , we obtain a value of $7.2 \mathrm{eV}$, which is similar to the value calculated for $\lambda_{\mathrm{exc}}=808 \mathrm{~nm}(1.53 \mathrm{eV})$, which yields $7.9 \mathrm{eV}$.

The calculated values of $E_{\text {tot }}$ for the wavelengths in the VIS region, i.e., $405 \mathrm{~nm}(3.06 \mathrm{eV}), 476 \mathrm{~nm}(2.60 \mathrm{eV}), 488 \mathrm{~nm}(2.54 \mathrm{eV})$ and $514 \mathrm{~nm}(2.41 \mathrm{eV})$, are $8.5 \mathrm{eV}, 7.9 \mathrm{eV}, 8.4 \mathrm{eV}$ and $8.9 \mathrm{eV}$, respectively.
The values of $E_{\text {tot }}$ calculated for the VIS excitation photons are higher than those for the infrared photons, which may be related to the different index of refraction for and better focusing of the visible light. The important observation is that regardless of the wavelength of the incident laser light used in the experiment, the total energy $E_{\text {tot }}$ of absorbed photons that gave rise to the WL emission was comparable and seems to have remained constant across the entire measured region.

This behavior may be well understood by considering the nearly frequency-independent absorption of graphene, which, for pristine graphene, is equal to the universal value of $\pi \alpha(2.29 \%)$ determined by the fine-structure constant $\alpha=e^{2} / \hbar c$. a

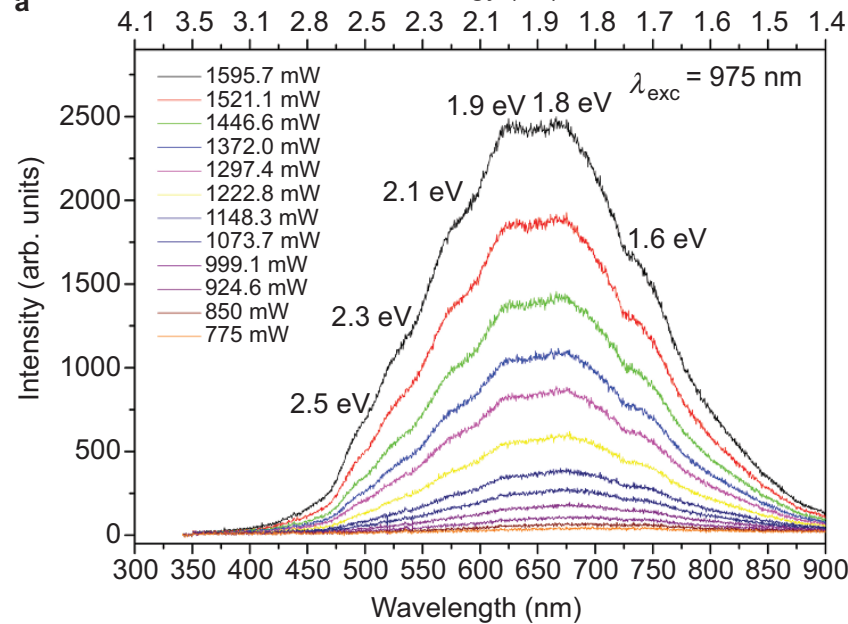

b



Figure 2 (a) Representative plot of laser-induced WL emission from graphene ceramics excited at $\lambda_{\text {exc }}=975 \mathrm{~nm}$ with various laser powers ranging from approximately $0.7 \mathrm{~W}$ up to approximately $1.5 \mathrm{~W}$. (b) The power dependence of the laser-induced WL emission at various excitation lines, i.e., $405 \mathrm{~nm}, 476 \mathrm{~nm}, 488 \mathrm{~nm}, 514 \mathrm{~nm}$, $808 \mathrm{~nm}$ and $975 \mathrm{~nm}$. The value of $N$ represents the order of the process. WL, white-light. 


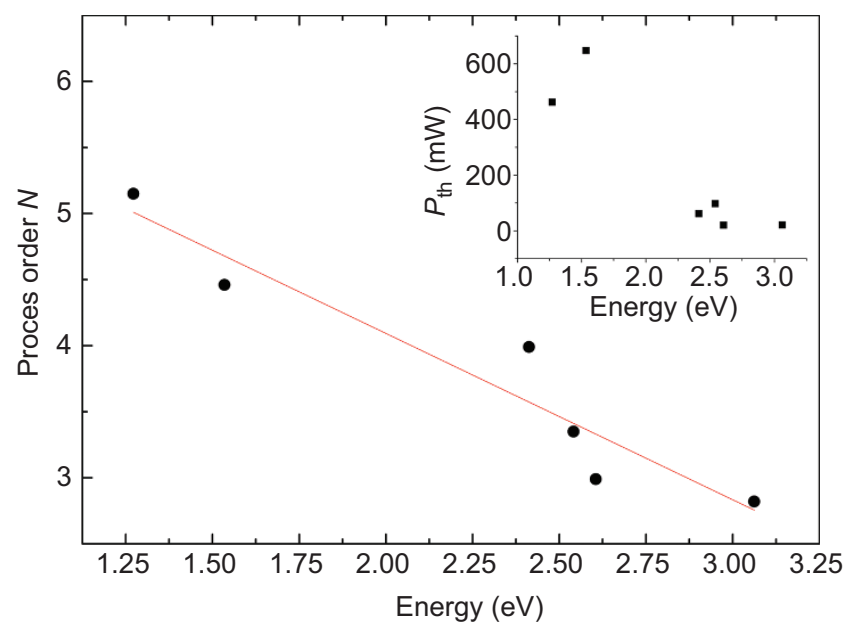

Figure 3 Dependence of the process order parameter $N$ on the excitation photon energy. The dependence of the threshold power $P_{\text {th }}$ on the photon excitation energy is presented in the inset.

A careful analysis of the power dependence of the WL emission revealed the threshold behavior of the WL emission. It was observed that $P_{\text {th }}$ decreased with increasing photon energy. The value of $P_{\text {th }}$ was at its lowest for blue-region photons (see the inset of Figure 3). This threshold behavior is characteristic of the multiphoton absorption processes responsible for photon avalanching and photoionization.

Because the WL emission from the graphene ceramics was observed only under a reduced atmospheric pressure, it was necessary to investigate the impact of the pressure conditions on the relative intensity of the WL emission. The results for the dependence on the ambient pressure are presented in Figure 4a. The intensity of the WL emission increased by a factor of approximately five upon the reduction of the pressure by over five decades to $5 \times 10^{-3} \mathrm{hPa}$ (Figure $4 \mathrm{~b}$ ). Although this increase is quite significant, much higher values have been observed in measurements of similar processes in lanthanide-doped dielectrics, e.g., $\mathrm{Yb}^{3+}: \mathrm{YAG}^{22}$ and $\mathrm{LiYbP}_{4} \mathrm{O}_{12}{ }^{23}$ nanocrystals. The dependence of the WL emission on the ambient pressure offers some clue as to the origin of the emission, which cannot simply be attributed to the contribution of functional groups, as for the emission of graphene oxide. The observed supralinear dependence of the intensity on the ambient pressure may be predicted by the model of the radiative dissipation of energy ${ }^{24}$ (Equation (2)):

$$
I_{\mathrm{em}}=I_{0} \exp \left(-\frac{P}{P_{0}}\right)
$$

where $P$ is the measured pressure and $I_{0}$ is the initial intensity at pressure $P_{0}$. It is clear that because of the observed saturation region, the results remain in agreement with this model; however, the lower dependence on the ambient pressure suggests that the convection process does not impact the emission intensity of our graphene ceramics as strongly as is observed in the dielectrics $\mathrm{Yb}^{3+}$ :YAG and $\mathrm{LiYbP}_{4} \mathrm{O}_{12}$.

The theory of black-body radiation is naturally the first choice for the interpretation of the origin of the WL emission in graphene. Although the nature of the photoluminescence bands is in conflict with that of black-body radiation, the question of the effective temperature of the sample is still one of fundamental interest. As can be calculated based on Planck's theorem and the characteristics of the WL emission, the temperature of the sample is expected to be approximately $4300 \mathrm{~K}$. Moreover, according to the theorem, a blue-shift of the emission band $\lambda_{\max }$ should be observed with increasing sample temperature. However, as was previously demonstrated by the powerdependence measurements, no significant spectral shift was observed. This raises a question regarding the true temperature of the emitting sample.

The temperature of the emitting graphene ceramics was measured using the $\mathrm{Er}^{3+}$-doped $\mathrm{LiYb}_{0.99} \mathrm{Er}_{0.01} \mathrm{P}_{4} \mathrm{O}_{12}$ upconverting nanocrystalline sensors encrusted on the surface of the emitting sample. The temperature sensing was achieved based on the well-known phenomenon that the ratio of the ${ }^{4} \mathrm{~S}_{3 / 2} \rightarrow{ }^{4} \mathrm{I}_{15 / 2}$ and ${ }^{2} \mathrm{H}_{11 / 2} \rightarrow{ }^{4} \mathrm{I}_{15 / 2}$ transitions is related to the change in temperature. The emission intensity from the higher-lying state ${ }^{2} \mathrm{H}_{11 / 2}$ depends on its population, which increases with temperature following the Boltzmann relation (Equation (3)):
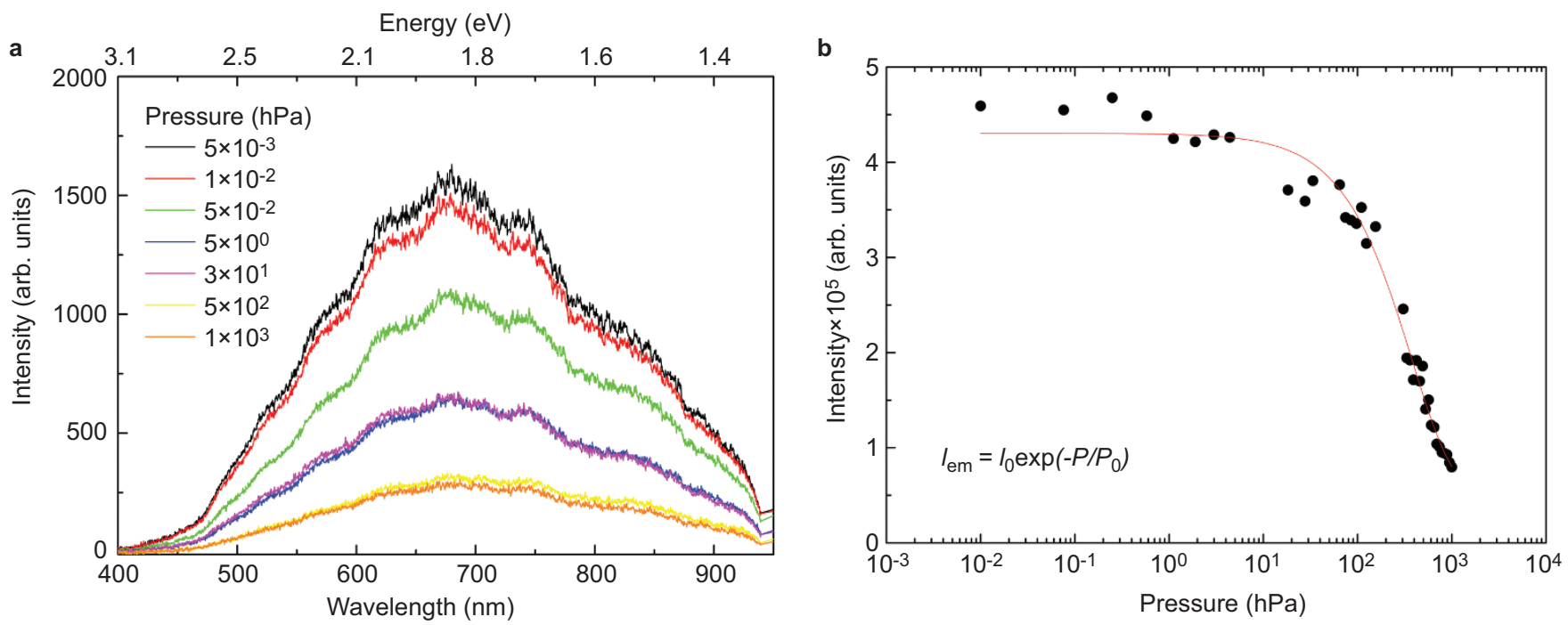

Figure 4 (a) Laser-induced WL emission spectra from graphene ceramics measured at various pressures and (b) the pressure dependence of the WL emission intensity. WL, white-light. 


$$
\frac{{ }^{I_{2}} \mathrm{H}_{11 / 2}}{{ }^{I_{4} \mathrm{~S}_{3 / 2}}}=A \cdot \exp \left(-\frac{\Delta E}{k_{\mathrm{B}} T}\right)+B
$$

where $A$ and $B$ are proportionality parameters, $\Delta E$ is the energy difference between the indicated states, $T$ is the temperature and $k_{\mathrm{B}}$ is the Boltzmann constant. The energy gap between the states in the case of $\mathrm{LiYb}_{0.99} \mathrm{Er}_{0.01} \mathrm{P}_{4} \mathrm{O}_{12}$ is approximately $810 \mathrm{~cm}^{-1}$. The sample to be measured was placed in vacuum $\left(5 \times 10^{-3} \mathrm{hPa}\right)$, and the upconversion emission spectra were recorded (Figure $5 \mathrm{a}$ ). Because the logarithm of the emission intensity ratio of ${ }^{4} \mathrm{~S}_{3 / 2} \rightarrow{ }^{4} \mathrm{I}_{15 / 2}$ to ${ }^{2} \mathrm{H}_{11 / 2} \rightarrow{ }^{4} \mathrm{I}_{15 / 2}$ is a linear function of temperature, by using the Boltzmann law, one is able to determine the temperature of nanocrystals subjected to laser excitation. Figure 5a presents the upconversion emission spectra of the $\mathrm{LiYb}_{0.99} \mathrm{Er}_{0.01} \mathrm{P}_{4} \mathrm{O}_{12}$ nanocrystals encrusted on the graphene ceramic surface.

Three characteristic $f-f$ fluorescence transitions of $\mathrm{Er}^{3+}$ located within the broadband WL emission of the graphene ceramics are clearly observed. For the low-optical-power regime, only the $\mathrm{Er}^{3+}$ fluorescence signatures localized near approximately $520 \mathrm{~nm}, 540 \mathrm{~nm}$ and $650 \mathrm{~nm}$ were observed; these signatures are associated with the ${ }^{2} \mathrm{H}_{11 / 2} \rightarrow{ }^{4} \mathrm{I}_{15 / 2}$, ${ }^{4} \mathrm{~S}_{3 / 2} \rightarrow{ }^{4} \mathrm{I}_{15 / 2}$ and ${ }^{4} \mathrm{~F}_{9 / 2} \rightarrow{ }^{4} \mathrm{I}_{15 / 2}$ transitions, respectively. With an increase in excitation power, the intensities of the UC transitions also increased. The results revealed that even for a very high excitation power of $1.5 \mathrm{~W}$ at $\lambda_{\text {exc }}=975 \mathrm{~nm}$, the temperature of the sample remained lower than $900 \mathrm{~K}$, and for excitation powers lower than $1 \mathrm{~W}$, the temperature was lower than $700 \mathrm{~K}$.

To determine whether the WL emission from the graphene ceramics could also be observed at lower temperatures, additional experiments were performed in a closed-cycle cryostat. The results are presented in Figure 5b. No particular temperature dependence was observed; indeed, the WL emission intensity remained nearly constant across a wide range of temperatures, from $10 \mathrm{~K}$ to $300 \mathrm{~K}$. It should be noted that similar experiments performed to investigate the anti-Stokes WL emission induced by an infrared laser in a dielectric host in the form of $\mathrm{LiNdP}_{4} \mathrm{O}_{12}$ nanocrystals ${ }^{22}$ have demonstrated clear evidence of temperature dependence, with complete quenching of the emission at very low temperatures.
These results provide a very compelling reason to reconsider the interpretation of the origins of laser-induced WL emission from graphene.

The photoelectrical response of the graphene ceramics provides additional information regarding the origin of the WL emission. The time-dependent photoelectric response of the graphene ceramics for 975-nm laser excitation at various powers is presented in Figure 6a. A linear dependence of the change in resistivity $\Delta \rho$ on the excitation power was observed, with values up to $\Delta \rho \approx 0.5 \mathrm{~m} \Omega \mathrm{cm}$. The timedependent characteristics of the process for two representative wavelengths of $\lambda_{\text {exc }}=405 \mathrm{~nm}$ and $975 \mathrm{~nm}$ are depicted in Figure $6 \mathrm{~b}$.

The photo-effect associated with the interband transition is pronounced for $405 \mathrm{~nm}$ excitation; however, thermo-electric effects contribute to the process, as is observed from the long saturation tail. The thermopower values of graphene ceramics may differ among samples, depending on their preparation temperatures; at $300 \mathrm{~K}$, these values may change from $6 \mu \mathrm{V} \mathrm{K}^{-1}$ to $20 \mu \mathrm{V} \mathrm{K} \mathrm{K}^{-1}$. A curve with a somewhat different character was observed for the $975-\mathrm{nm}$ excitation wavelength. Pronounced effects of thermal processes were also clearly observed. None of the curves was observed to follow the first-order rate law, implying that more than one process was involved in the change in resistivity. The time constants of the processes under 975-nm excitation were estimated to be $\tau_{1}=10.8 \mathrm{~s}$ and $\tau_{2}=63.2 \mathrm{~s}$.

There are several considerations that may be useful in forming any conclusions to be drawn regarding the origin of the WL emission from graphene ceramics. Because pristine graphene has no energy gap, no radiative emission is observed under typical conditions of optical excitation. On the other hand, emission from thermalized electrons-incandescence-requires temperatures much higher than those measured in this work. It was also demonstrated that the WL emission is a threshold process, which suggests that emission from graphene may be observed after a certain energy barrier is crossed (in our experiments, $E_{\text {tot }}=N_{\text {exc }} \hbar \omega_{\text {exc }} \approx 8 \mathrm{eV}$ ), leading to the creation of transient, non-equilibrium phases with a different distribution of charge and a different electronic structure. It has previously been demonstrated that non-equilibrium states in graphene involving a population of inverted dense Dirac fermions emerge during femtosecond pulse propagation. ${ }^{25}$ This observation supports the concept of photoinduced transitions of $\mathrm{sp}^{2} \rightarrow \mathrm{sp}^{3}$ hybridization in disordered
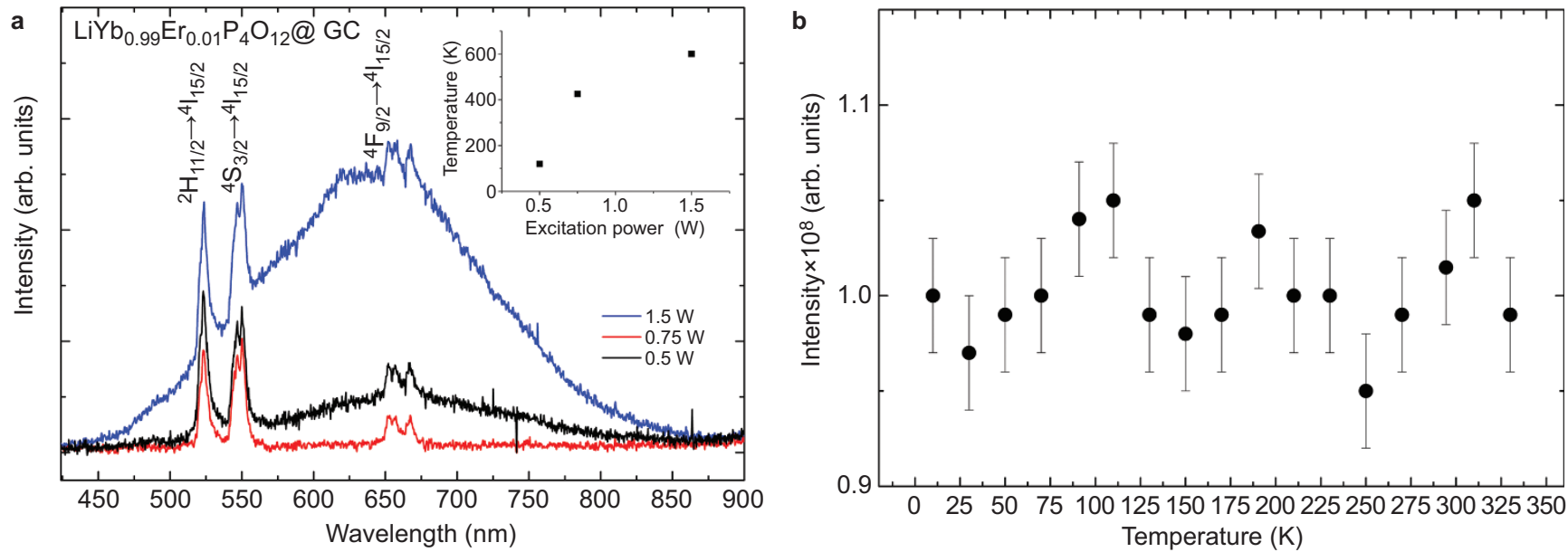

Figure 5 (a) WL emission of graphene ceramics encrusted with $\mathrm{LiYb}_{0.99} \mathrm{Er}_{0.01} \mathrm{P}_{4} \mathrm{O}_{12}$ upconverting nanoparticles excited by a 975 -nm $\mathrm{CW}$ laser at various pumping powers. The upconversion emission bands from $\mathrm{Er}^{3+}$ are clearly evident in the broad emission of the graphene sample. The temperature of the sample was calculated using Equation (3). The $A$ and $B$ parameters were determined to be 0.00386 and -1.44 , respectively. A plot of the effective temperature of the sample as a function of the optical excitation power is presented in the inset. (b) Dependence of the WL intensity on the sample temperature. CW, continuous-wave; GC, graphene ceramic; WL, white-light. 

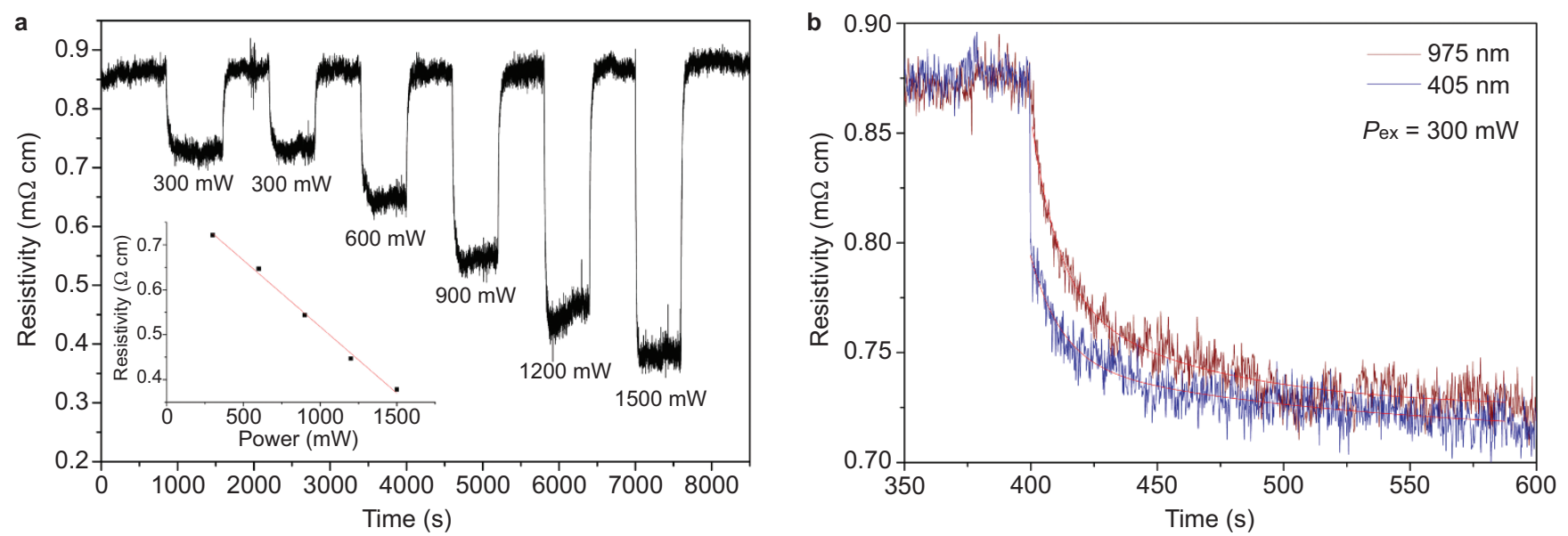


$\lambda_{\text {exc }}=975 \mathrm{~nm}$. (b) Time-dependent characteristics of the resistivity after exposure to $300-\mathrm{mW}$ laser beams at $\lambda_{\mathrm{exc}}=405 \mathrm{~nm}$ and $975 \mathrm{~nm}$.

phases such as graphene ceramics. Several studies have been published concerning light emission under femtosecond light stimulation. ${ }^{26}$ However, the first reports of CW-excited hot luminescence were published by Chen et al., ${ }^{27}$ who were studying inelastic light scattering in highly doped graphene; WL emission from cold samples has never previously been reported. It is also worth noting that photoinduced phase transitions involving switching between anti-ferromagnetic and magnetic states induced by femtosecond pulses via strongly correlated spin-charge quantum excitation have also been demonstrated by $\mathrm{Li}$ et al. ${ }^{28}$ in non-carbonaceous compounds $\left(\mathrm{Pr}_{0.7} \mathrm{Ca}_{0.3} \mathrm{MnO}_{3}\right)$.

Previous attempts to open a band gap in graphene have concentrated on the physical modification of the material, in which delocalization of $\pi$ bonds induces the creation of the band gap. ${ }^{29,30}$ In this work, we propose a model of photoinduced phase transformation to explain the WL emission from CW-excited graphene ceramics. ${ }^{31}$ The photoinduced transition process relies on a hidden multistability of the material, meaning that in addition to the true ground state, there are also hidden, so-called false ground states that are inaccessible through thermal fluctuations but achievable through electronic excitation. A conceptual diagram of the photoinduced transition process is presented in Figure 7.

Recent theoretical ${ }^{32-35}$ and experimental ${ }^{36,37}$ results have indicated the existence of a new stable phase of carbon with mixed $\mathrm{sp}^{2}$ and $\mathrm{sp}^{3}$ hybridized bonds - diaphite. In one such experiment, highly oriented pyrolytic graphite was excited using a femtosecond laser (1.57-eV photon energy), leading to electron transfer between the $\pi$ and $\pi^{*}$ bands. Similar effects may also be induced via CW excitation, with two or three photons inducing a charge transfer from the $\pi$ band to the $\pi^{*}$ band and creating an electron-hole pair. ${ }^{35,38}$ A conceptual diagram of this mechanism is presented in Figure 8. The latter process leads to the creation of transient localized states of electron-hole pairs. The electron-optical phonon coupling leads to graphene lattice contraction, whereas unlocalized states contribute to the conduction band. Henceforth, a small amount of electronic energy is converted into kinetic energy of the lattice, leading to the heating of the material. The overall process is a multiphoton process; however, as shown in Refs. 34, 39 and 40, a minimal diaphite domain may be created when the energy deposited in the system exceeds $4.5 \mathrm{eV}$, which corresponds to only a few visible photons. The transformation is nonlinear because of the strong coupling between neighboring atoms in graphite sheets. It is worth noting that the resulting new phase-diaphite-has already been observed to be a quasistable structure, with stability of up to a few days. ${ }^{36}$ As a result of the $\mathrm{sp}^{2} \rightarrow \mathrm{sp}^{3}$ transition, a band gap opens, resulting in the creation of a semiconducting phase immersed in the semimetallic continuum. Although this process has been observed in graphite, the theoretical interpretation presented suggests that similar behavior should occur in all carbon systems with a linear band shape in the visible energy regime. Henceforth, the observation of phenomena of this type is to be expected in bi- and trilayer graphene, as well.

Our analysis suggests that the observed emission is purely electronic in nature. This conclusion explains both the heating of the material (energy transfer via free photo-generated carrier-phonon coupling) and the power-law dependence (threshold energy required for diaphite domain formation). The remaining majority of unlocalized carriers will contribute to the ionization process. As stated above, $\mathrm{CW}$ excitation will lead to two types of carriers-localized and unlocalized. Coulomb repulsion will induce rapid separation from the excited domain to the metallic continuum (Figure 8). As a result, the remaining carbon atoms will have a lower ionization potential because of the appearance of excess charge in the system and $\pi$-bond expansion.

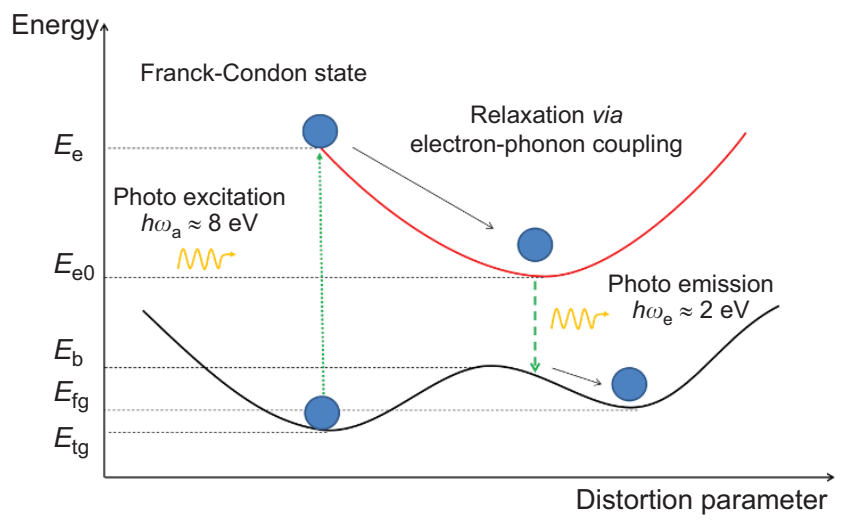

Figure 7 Schematic illustration of photoinduced structural phase transformation with one photon excitation. The system consists of a true ground state $\left(E_{\mathrm{tg}}\right)$ and a false ground state $\left(E_{\mathrm{fg}}\right)$ separated by an energy barrier higher than the thermal fluctuations $\left(E_{\mathrm{b}}-E_{\mathrm{tg}}>k T\right)$. The electron is vertically excited by a photon $\left(h \omega_{\mathrm{a}}\right)$ to an excited state of energy $E_{\mathrm{e}}$. Via electron-phonon coupling, the electron relaxes non-radiatively to the excited energy surface minimum $\left(E_{\mathrm{e} 0}\right)$, and it then recombines radiatively by emitting a photon $\left(h \omega_{\mathrm{e}}<h \omega_{\mathrm{a}}\right)$. 


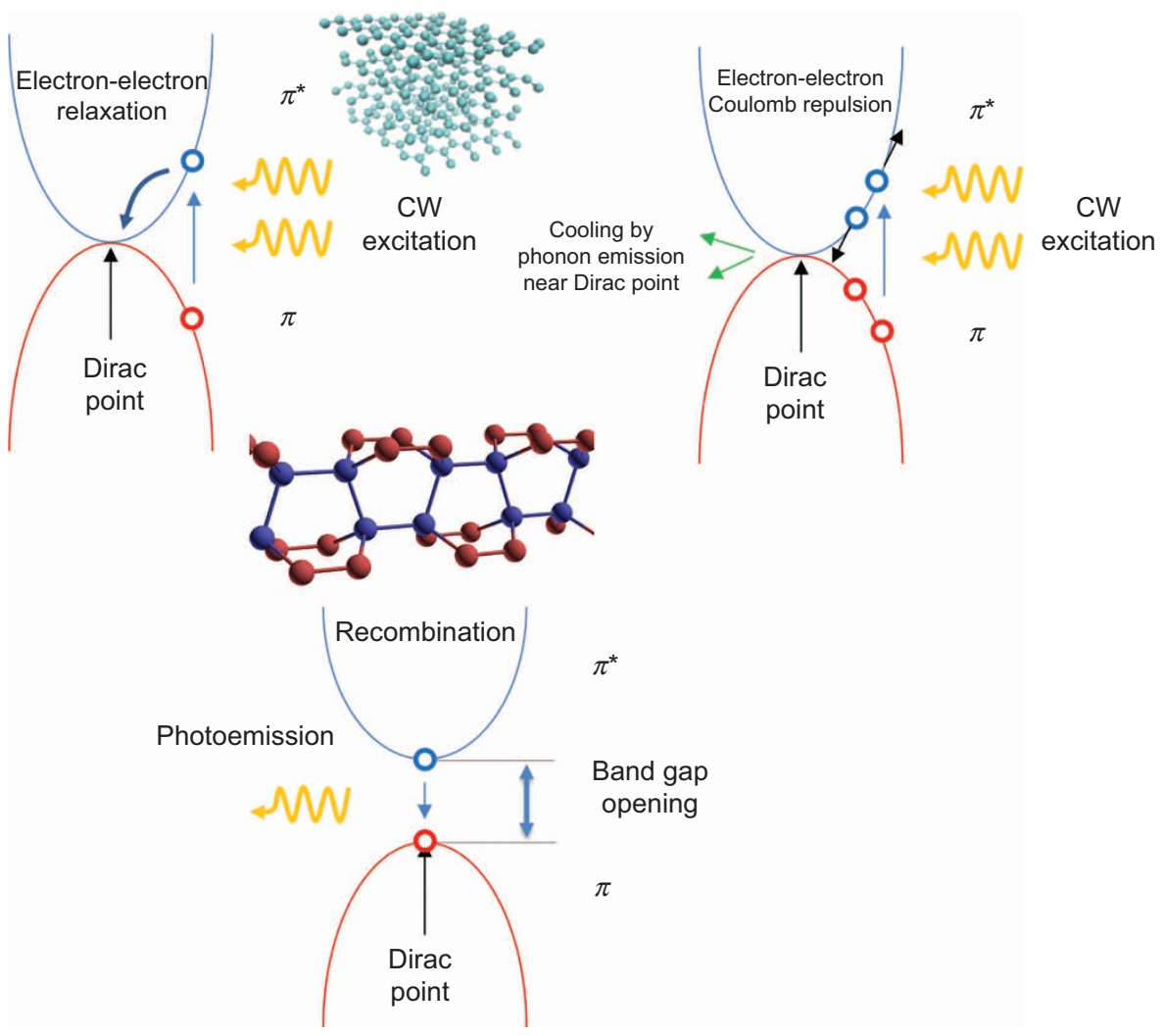

Figure $8 \mathrm{CW}$ excitation models in bilayer and trilayer graphene systems. Initially, graphene is a zero-gap semiconductor. Photoexcitation promotes an electron from the $\pi$ band to the $\pi^{*}$ band, creating an electron-hole pair. Subsequent excitations create a growing population of mutually repulsive electron-hole states. The electronic energy is then directed into electron-hole localization and diaphite domain creation. As a result of the $\mathrm{sp}^{2} \rightarrow \mathrm{sp}^{3}$ transition, a band gap opens, enabling photoluminescence processes. CW, continuous-wave.

Because the ionization potential in graphene is approximately $8 \mathrm{eV}$, multiphoton direct ionization will occur. Photoinduced carriers will recombine with ionized carbons or contribute to the conduction $\pi$ band, thereby lowering the ionization potential.

\section{CONCLUSIONS}

Laser-induced WL emission from graphene ceramics was investigated in this work. Intense WL emission centered at $650 \mathrm{~nm}$ and consisting of a number of subbands was observed. No significant dependence of the broadband WL emission maximum on the excitation wavelength was observed. Laser-induced WL emission was observed at a number of different excitation wavelengths, from the VIS to the NIR range, i.e., at $476 \mathrm{~nm}, 488 \mathrm{~nm}, 514 \mathrm{~nm}, 808 \mathrm{~nm}$ and $974 \mathrm{~nm}$. It was also observed that the WL emission from graphene ceramics was a threshold process exhibiting supralinear behavior. It was observed that the intensity of the laser-induced WL emission increased nonlinearly with increasing incident laser power, with a strong increase in intensity after a certain power threshold that could be well fitted using a power law, $P^{N}$. The order of the process, $N$, decreased with increasing frequency of the incident laser light. Similarly, the excitation power threshold decreased with increasing frequency of the incident laser light. The total energy $E_{\text {tot }}$ of $N$ absorbed photons with energy $\hbar \omega_{\text {exc }}$ remained nearly constant for all investigated excitation wavelengths, $E_{\text {tot }}=N_{\text {exc }} \hbar \omega_{\text {exc }} \approx 8 \mathrm{eV}$. It was also demonstrated that the intensity of the WL emission was highly dependent on the ambient pressure. The highest intensity was observed at the lowest pressure.

The temperatures of the samples were also measured using $\mathrm{LiYb}_{0.99}$ $\mathrm{Er}_{0.01} \mathrm{P}_{4} \mathrm{O}_{12}$ upconverting nanocrystals encrusted on the surfaces of the graphene ceramics. The results revealed that even for a very high excitation power of $1.5 \mathrm{~W}$ at $\lambda=975 \mathrm{~nm}$, the temperature of the sample remained lower than $900 \mathrm{~K}$, and for excitation powers lower than $1 \mathrm{~W}$, the temperature was below $700 \mathrm{~K}$. No dependence of the WL emission intensity on the sample temperature was observed, and WL emission was also observed at cryogenic temperatures as low as $10 \mathrm{~K}$. This result provides compelling evidence that even when the sample is hot, its temperature is still far too low to explain the emission according to the theory of thermalized electrons. Efficient photocurrent was observed in the graphene ceramics. It increased linearly with increasing power of the incident laser light. A model of photoinduced, transient, domain-like $\mathrm{sp}^{2} \rightarrow \mathrm{sp}^{3}$ phase transitions was proposed to explain the origin of the multiphoton-induced WL emission in graphene ceramics.

\section{ACKNOWLEDGEMENTS}

This work was supported by Wroclaw Research Centre EIT+ within the framework of the project 'The Application of Nanotechnology in Advanced Materials'-NanoMat (POIG.01.01.02-02-002/08) financed by the European Regional Development Fund (Operational Programme Innovative Economy, 1.1.2).

1 Geim AK, Novoselov KS. The rise of graphene. Nat Mater 2007; 6: 183-191.

2 Schwierz F. Graphene transistors. Nat Nanotechnol 2010; 5: 487-496.

3 Chen MT, Tao T, Zhang L, Gao W, Li CZ. Highly conductive and stretchable polymer composites based on graphene/MWCNT network. Chem Commun 2013; 49: 16121614 
4 Liu YX, Dong $X C$, Chen P. Biological and chemical sensors based on graphene materials. Chem Soc Rev 2012; 41: 2283-2307.

5 He QY, Wu SX, Yin ZY, Zhang H. Graphene-based electronic sensors. Chem Sci 2012; 3: 1764-1772.

6 Loh KP, Bao QL, Eda G, Chhowalla M. Graphene oxide as a chemically tunable platform for optical applications. Nat Chem 2010; 2: 1015-1024.

7 Li M, Cushing SK, Zhou XJ, Guo SW, Wu NQ. Fingerprinting photoluminescence of functional groups in graphene oxide. J Mater Chem 2012; 22: 23374-23379.

8 de Volder MF, Tawfick SH, Baughman RH, Hart AJ. Carbon nanotubes: present and future commercial applications. Science 2013; 339: 535-539.

9 Dresselhaus MS, Dresselhaus G. Fullerenes and fullerene-derived solids as electronic materials. Annu Rev Mater Sci 1995; 25: 487-523.

10 Guldi DM, Illescas BM, Atienza CM, Wielopolski M, Martín N. Fullerene for organic electronics. Chem Soc Rev 2009; 38: 1587-1597.

11 Novoselov KS, Fal'ko VI, Colombo L, Gellert PR, Schwab MG et al. A roadmap for graphene. Nature 2012; 490: 192-200.

12 Castro Neto AH, Guinea F, Peres NM, Novoselov KS, Geim AK. The electronic properties of graphene. Rev Mod Phys 2009; 81: 109-162.

13 Yu DS, Dai LM. Voltage-induced incandescent light emission from large-area graphene films. Appl Phys Lett 2010; 96: 143107.

14 Scarselli M, Castrucci P, de Crescenzi M. Electronic and optoelectronic nano-devices based on carbon nanotubes. J Phys Condens Mat 2012; 24: 313202.

15 Mak KF, Ju L, Wang F, Heinz TF. Optical spectroscopy of graphene: from the far infrared to the ultraviolet. Solid State Commun 2012; 152: 1341-1349.

16 Ferrari AC, Meyer JC, Scardaci V, Casiraghi C, Lazzeri M et al. Raman spectrum of graphene and graphene layers. Phys Rev Lett 2006; 97: 187401.

17 Ikezawa S, Wakamatsu M, Ueda T. Optical analytical technique for carbonaceous particles using laser-induced electro-avalanche fluorescence and laser-induced incandescence. Sensor Mater 2013; 25: 57-77.

18 Essig S, Marquardt CW, Vijayaraghavan A, Ganzhorn M, Dehm S et al. Phonon-assisted electroluminescence from metallic carbon nanotubes and graphene. Nano Lett2010; 10: 1589-1594.

19 Biswas C, Güneș F, Loc DD, Lim SC, Jeong MS et al. Negative and positive persistent photoconductance in graphene. Nano Lett 2011; 11: 4682-4687.

20 Dong YQ, Shao JW, Chen CQ, Li H, Wang RX et al. Blue luminescent graphene quantum dots and graphene oxide prepared by tuning the carbonization degree of citric acid. Carbon 2012; 50: 4738-4743.

21 Sekiya R, Uemura Y, Murakami H, Haino T. White-light-emitting edge-functionalized graphene quantum dots. Angew Chem Int Ed 2014; 53: 5619-5623.

22 Strek W, Marciniak L, Gluchowski P, Hreniak D. Infrared laser stimulated broadband white emission of $\mathrm{Yb}^{3+}$ :YAG nanoceramics. Opt Mater 2013; 35: 2013-2017.

23 Strek W, Marciniak L, Bednarkiewicz A, Lukowiak A, Wiglusz R et al. White emission of lithium ytterbium tetraphosphate nanocrystals. Opt Express 2011; 19: 1408314092.

24 Wang JW, Tanner PA. Upconversion for white light generation by a single compound. J Am Chem Soc 2010; 132: 947-949.

$25 \mathrm{Li} \mathrm{T,} \mathrm{Luo} \mathrm{L,} \mathrm{Hupalo} \mathrm{M,} \mathrm{Zhang} \mathrm{J,} \mathrm{Tringides} \mathrm{MC} \mathrm{et} \mathrm{al.} \mathrm{Femtosecond} \mathrm{population} \mathrm{inversion}$ and stimulated emission of dense Dirac fermions in graphene. Phys Rev Lett 2012; 108: 167401 .
26 Lui CH, Mak KF, Shan J, Heinz TF. Ultrafast photoluminescence from graphene. Phys Rev Lett 2010; 105: 127404.

27 Chen CF, Park CH, Boudouris BW, Horng J, Geng B et al. Controlling inelastic light scattering quantum pathways in graphene. Nature 2011; 471: 617-620.

28 Li T, Patz A, Mouchliadis L, Yan J, Lograsso TA et al. Femtosecond switching of magnetism via strongly correlated spin-charge quantum excitations. Nature 2013; 496: 69-73.

29 Han MY, Özyilmaz B, Zhang YB, Kim P. Energy band-gap engineering of graphene nanoribbons. Phys Rev Lett 2007; 98: 206805.

30 Shen Y, Yang SB, Zhou P, Sun QQ, Wang PF et al. Evolution of the band-gap and optical properties of graphene oxide with controllable reduction level. Carbon 2013; 62: 157-164.

31 Huai P, Nasu K. Theory of photoinduced phase transition in the quasi-onedimensional charge transfer compound TTF-CA. Int J Mod Phys B 2001; 15: 37143717.

32 Ohnishi H, Nasu K. Photoinduced domain-type collective structural changes with interlayer sigma-bonds in the visible region of graphite. Phys Rev B 2009; 79: 054111.

33 Ohnishi H, Nasu K. Generation and growth of $s p^{3}$ bonded domains by visible photon irradiation of graphite. Phys Rev B 2009; 80: 014112.

34 Nishioka K, Nasu K. Early-stage real-time dynamics of interlayer $s p^{3}$ bond formation by visible-light irradiation of graphite. Phys Rev B 2009; 80: 235420.

35 Radosinski L, Nasu K, Luty T, Radosz A. Possible domain-type collective dimerization of graphite induced by interlayer charge transfer excitations in the visible region. Phys Rev B 2010; 81: 035417.

36 Kanasaki J, Inami E, Tanimura K, Ohnishi H, Nasu K. Formation of $s p^{3}$ bonded carbon nanostructures by femtosecond laser excitation of graphite. Phys Rev Lett2009; 102: 087402.

37 Raman RK, Murooka Y, Ruan CY, Yang T, Berber S et al. Direct observation of optically induced transient structures in graphite using ultrafast electron crystallography. Phys Rev Lett 2008; 101: 077401.

38 Radosinski L, Ostasiewicz K, Luty T, Radosz A, Nasu K. Domain-type collective dimerization of graphite and possible $s p^{2} \rightarrow s p^{3}$ transition induced by inter-layer charge transfer excitations in the visible region. Acta Phys Pol A 2010; 118: 500506

39 Radosinski L, Luty T, Nasub K, Ohnishi H, Nishioka K et al. Photoinduced conversion of hybridization in graphite. Acta Phys Pol A 2012; 121: 359-368.

40 Nishioka K, Nasu K. Cooperative domain-type interlayer $s p^{3}$ bond formation in graphite. Phys Rev B 2010; 82: 035440.

\section{(c) (i) (2) This work is licensed under a Creative Commons Attribution-}

NC SA NonCommercial-ShareAlike 3.0 Unported License. The images or other third party material in this article are included in the article's Creative Commons license, unless indicated otherwise in the credit line; if the material is not included under the Creative Commons license, users will need to obtain permission from the license holder to reproduce the material. To view a copy of this license, visit http://creativecommons.org/licenses/by-nc-sa/3.0/ 